Knie J. $2020 \cdot 2: 157-158$

https://doi.org/10.1007/s43205-020-00075-8

(c) Springer Medizin Verlag GmbH, ein Teil von Springer Nature 2020

\author{
Mirco Herbort ${ }^{1,2} \cdot$ Andrea Achtnich $^{3} \cdot$ Karl-Heinz Frosch $^{4,5}$ \\ 'OCM-Klinik, München, Deutschland \\ ${ }^{2}$ Forschungsbereich orthopädische Sportmedizin und Verletzungsprävention, UMIT, Hall, Österreich \\ ${ }^{3}$ Klinikum rechts der Isar, Abteilung für Sportorthopädie, TU München, München, Deutschland \\ ${ }^{4}$ Klinik für Unfallchirurgie und Orthopädie, Universitätsklinikum Hamburg-Eppendorf, Hamburg, \\ Deutschland \\ ${ }^{5}$ Abteilung Unfallchirurgie, Orthopädie und Sporttraumatologie, BG Klinikum Hamburg, Hamburg, \\ Deutschland
}

\title{
Stellenwert der peripheren Instabilitäten des Kniegelenkes
}

kenden Kräften zu widerstehen und somit das Kniegelenk gegen Rotationsund Varus-/Valgusstress zu stabilisieren.

Die synergistisch stabilisierende Bedeutung der medialen und lateralen Gelenkecke für die Versorgung der hinteren Kreuzbandverletzungen ist bereits seit langer Zeit bekannt. So wird eine ausgeprägte hintere Schublade auf eine Kombinationsverletzung des hinteren Kreuzbandes mit den posterolateralen oder posteromedialen Strukturen zurückgeführt $[3,6]$. Um diese hochgradige Instabilität erfolgreich behandeln $\mathrm{zu}$ können, gilt die kombinierte operative Versorgung des hinteren Kreuzbandes zusammen mit den Seitenbandstrukturen als Versorgungsstandard. Insbesondere in der chronischen Situation sind die begleitenden Seitenbandinstabilitäten nicht einfach zu diagnostizieren [4].

\section{》) In der chronischen Situation sind begleitende Seitenbandinstabilitäten schwer zu diagnostizieren} vollziehbar. Die Hebelbedingungen von $\mathrm{VKB} / \mathrm{HKB}$, welche im Zentrum des Kniegelenkes angeordnet sind, sind eher ungünstig zur Stabilisierung gegen diese Rotations- und Varus-/Valguskräfte. Die peripheren Bandstrukturen mit ihrem verbesserten Kraft-/Lastarmverhältnis hinsichtlich Rotationskräften bieten mit ihrem anatomischen Verlauf hingegen ideale Voraussetzungen, diesen einwir- nahmen das entscheidende Kriterium zur Analyse der gesamten Verletzung und die Voraussetzung einer adäquaten operativen Versorgungsplanung [4].

In den letzten Jahren ist dieser synergistische Stabilisierungseffekt durch die peripheren Bandstrukturen im Falle einer VKB-Ruptur zunehmend in das Bewusstsein geraten.

Bei der genauen Analyse von VKBRezidivinstabilitäten und Rerupturen haben sich vermehrte Rotationsinstabilitäten als wichtiger Parameter für die Standzeit des Transplantates herausgestellt.

Insbesondere die vermehrte anterolaterale Rotationsinstabilität, die sich in unterschiedlichem Ausmaß nach erlittener VKB-Ruptur feststellen lässt, scheint offensichtlich einen großen Einfluss auf die Ergebnisse der vorderen Kreuzbandchirurgie zu haben. Allein die Tatsache, dass nach einer vollständigen VKB-Ruptur die resultierende Rotationinstabilität (ermittelt durch das Ausmaß des Pivot-Shiftes) sehr unterschiedlich ausfällt, muss als ein Hinweis auf eine notwendige Begleitpathologie oder -verletzung gewertet werden. Lange Zeit war jedoch eine isolierte, möglichst anatomische Rekonstruktion des vorderen Kreuzbandes (z. B. Doppelbündelrekonstruktion des VKB) der alleinige Therapieansatz zur Behandlung einer anterolateralen Knieinstabilität und des daraus resultierenden Pivot-Shift-Effektes.

Klinische Studien über die Ergebnisse von Revisionsrekonstruktionen zeigten jedoch zum größten Teil enttäuschen- 
de klinische Ergebnisse und eine relative hohe Anzahl von verbleibenden Rotationsinstabilitäten, was den Schluss zulässt, dass eine isolierte Rekonstruktion des zentralen Pfeilers nicht ausreichend ist, um diese komplexe Instabilität erfolgreich zu behandeln $[1,2]$.

Die Bemühungen in den vergangenen 10-15 Jahren durch eine isolierte Modifikation der VKB-Rekonstruktionstechnik (z.B. Doppelbündelrekonstruktion) diese hochgradigen Rotationsinstabilitäten (Pivot-Shift Grad 2-3) in den Griff zu bekommen, waren leider nicht in allen Fällen von Erfolg gekrönt. Zusätzlich wurde in der Vergangenheit die unterschiedliche Ausprägung der Instabilität nach „vermeintlich isolierter VKB-Ruptur" unkritisch in Kauf genommen und mithilfe einer isolierten VKB-Rekonstruktion behandelt. Nach genauer Betrachtung dieser Problematik erscheint es logisch, dass Begleitpathologien (traumatisch oder auch habituell) für diese unterschiedliche Ausprägung der Instabilität nach VKB-Ruptur verantwortlich sein können [5, 7].

Die am häufigsten vorkommende Rotationsinstabilität nach Ruptur des vorderen Kreuzbandes ist die anterolaterale Rotationsinstabilität. Diese wird unter anderem in Zusammenhang mit Verletzungen der anterolateralen Kapselbandstrukturen gebracht. Die Diskussion über die „Wiederentdeckung“ des anterolateralen Ligamentes (ALL) hat den Fokus auf diese Rotationsinstabilität gelenkt.

Neben der anterolateralen Rotationsinstabilität gibt es jedoch auch gehäuft eine anteromediale Rotationsinstabilität, welche durch eine (Teil-)Insuffizienz der (postero-)medialen Bandstrukturen verursacht wird [7]. Diese anteromediale Rotationsinstabilität führt zu einer vermehrten pathologischen Außenrotationsfähigkeit des Unterschenkels, welche durch die Insuffizienz des (postero-)medialen Bandapparates verursacht wird [7]. Diese Begleitinstabilität kann offensichtlich ein wichtiger Grund für das Versagen einer isolierten VKB-Rekonstruktion sein [1].

Somit ist es sehr wichtig, zum einen zwischen Innen- und Außenrotationsinstabilitäten insbesondere im Zusammenhang mit VKB-Rupturen zu unterschei- den und diese durch eine dezidierte klinische Untersuchung zu erkennen und bei Bedarf auch zu adressieren [4].

\section{Zusammenfassung}

Die peripheren Bandstrukturen des Kniegelenkes haben einen wichtigen synergistisch stabilisierenden Einfluss auf die Bandstrukturen des zentralen Pfeilers (VKB/HBK). Aufgrund ihrer dezentralen Lage im Kniegelenk sind sie dem zentralen Pfeiler bei der Stabilisierung gegen Rotations- und Varus-/ Valguskräfte überlegen. Zur Vermeidung von Rezidivinstabilitäten sollten diese peripheren Bandstrukturen respektiert und bei Insuffizienz operativ adressiert werden. In den folgenden Übersichtsarbeiten dieser Ausgabe des Knie Journals soll dieser Themenkomplex detailliert in Bezug auf Diagnostik und operative Behandlung aufgearbeitet werden und einen Leitfaden für die tägliche Praxis darstellen.

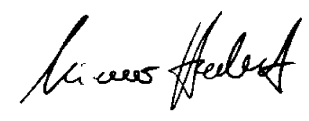

Prof. Dr. Mirco Herbort,

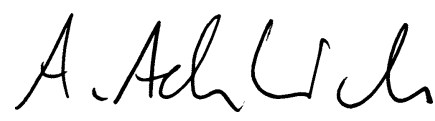

PD Dr. Andrea Achtnich,

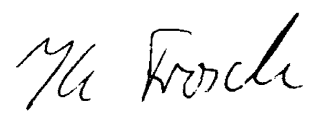

Univ.-Prof. Dr. Karl-Heinz Frosch

\section{Korrespondenzadresse}

Prof. Dr. Mirco Herbort
OCM-Klinik
Steinerstraße 6, 81369 Mün-
chen, Deutschland
Mirco.Herbort@
ocm-muenchen.de

Interessenkonflikt. M. Herbort, A. Achtnich und K.-H. Frosch geben an, dass kein Interessenkonflikt besteht.

\section{Literatur}

1. Alm L, Krause M, Frosch K-H, Akoto R (2020) Preoperative medial knee instability is an underestimated risk factor for failure of revision $\mathrm{ACL}$ reconstruction. Knee Surg Sports Traumatol Arthrosc. https://doi. org/10.1007/s00167-020-06133-y

2. Anand BS, Feller JA, Richmond AK, Webster KE (2016) Return-to-sport outcomes after revision anterior cruciate ligament reconstruction surgery. Am J Sports Med 44:580-584

3. Domnick C, Frosch K-H, Raschke MJ, Vogel N, Schulze M, von Glahn M, Drenck TC, Herbort M (2017) Kinematics of different components of the posterolateral corner of the knee in the lateral collateral ligament-intact state: a human cadaveric study. Arthroscopy 33:1821-1830.e1

4. Herbort M, KittlC, DomnickC, Glasbrenner J, FinkC, Herbst E (2019) Clinical examination of the knee. Unfallchirurg 122:977-991

5. Kittl C, El-Daou H, Athwal KK, Gupte CM, Weiler A, Williams A, Amis AA (2016) The role of the anterolateral structures and the $\mathrm{ACL}$ in controlling laxity of the intact and ACL-deficient knee. Am J Sports Med 44:345-354

6. Weimann A, Schatka I, Herbort M, Achtnich A, ZantopT, RaschkeM,Petersen W(2012) Reconstruction of the posterior oblique ligament and the posterior cruciate ligament in knees with posteromedial instability. Arthroscopy 28:1283-1289

7. Wierer G, Milinkovic D, Robinson JR, Raschke MJ, Weiler A, Fink C, Herbort M, Kittl C (2020) The superficial medial collateral ligament is the major restraint to anteromedial instability of the knee. Knee Surg Sports Traumatol Arthrosc. https://doi. org/10.1007/s00167-020-05947-0 